Apuntes Universitarios, 2022: 12 (2), abril-junio

ISSN: 2304-0335 DOI: https://doi.org/10.17162/au.v12i2.1035

\title{
Inteligencia emocional como herramienta eficaz para la mejora del desempeño laboral en funcionarios públicos: Una revisión
}

\author{
Emotional intelligence as an effective tool for improving job performance \\ in public officials: A review
}

\author{
Elvia Margarita Diaz Ortiz; ${ }^{1 \mathrm{a}}$ Patricia del Rocio Chávarry Ysla ${ }^{\mathrm{b}}$ \\ Universidad César Vallejo, Chiclayo, Perú ${ }^{12}$ \\ ORCID ID: https://orcid.org/0000-0002-7181-6272 1 \\ ORCID ID: https://orcid.org/0000-0003-0575-3717²
}

Recibido: 02 de setiembre de 2021

Aceptado: 10 de diciembre de 2021

\section{Resumen}

La presente investigación tuvo por objetivo recopilar información en estudios desarrollados sobre la inteligencia emocional como herramienta eficaz para la mejora del desempeño laboral en funcionarios que prestan servicios en una organización. Se realizó una búsqueda en la base de datos de Scopus y ScienceDirect, y se seleccionaron 18 artículos originales, cuya antigüedad de publicación resulta de hace seis años. Los resultados ponen en evidencia que la inteligencia emocional aplicada como herramienta estratégica mejora el desempeño de los trabajadores dentro de los cargos que poseen; además, diversos estudios muestran una relación positiva entre inteligencia emocional y desempeño laboral. Finalmente, se concluye que la inteligencia emocional se ha posicionado como uno de los elementos más indispensables dentro del entorno laboral, pues no solo permite mantener motivado al personal, sino también mejora el clima laboral y faculta al funcionario interactuar saludablemente con los miembros la organización.

Palabras clave: inteligencia emocional; desempeño laboral; estrés laboral; estrategias de afrontamiento; servidor público.

\footnotetext{
Abstract

${ }^{a}$ Correspondencia al autor:

E-mail: elviamargaritadiazortiz@gmail.com
}

The purpose of this research was to gather information on studies developed on emotional intelligence as an effective tool for improving work performance in employees serving in an organization. A search was carried out in the Scopus and ScienceDirect databases, and 18 original articles were selected, which were published within the last six years. The results show that emotional intelligence applied as a strategic tool improves the performance of workers in the positions they hold; in addition, several studies show a positive relationship between emotional 
intelligence and job performance. Finally, it is concluded that emotional intelligence has positioned itself as one of the most indispensable elements within the work environment, since it not only keeps the personnel motivated, but also improves the work environment and enables the employee to interact healthily with the members of the organization.

Keywords: emotional intelligence; job performance; job stress; coping strategies; public servant.

\section{Introducción}

Es de suma urgencia dar respuesta a las necesidades y exigencias en las organizaciones a través de la creatividad, el liderazgo y el trabajo en equipo, con la finalidad de obtener la excelencia en los servicios brindados (Macht et al., 2019). En tal sentido, requiere enfatizar las competencias que un profesional debe poseer; es decir, tomar en cuenta aspectos blandos vinculados a la inteligencia emocional, competencias que van más allá del tradicional enfoque centrado en el cúmulo de información y procesos operativos, para dar pase a un perfil que se caracteriza por la flexibilidad de pensamiento y la comprensión de sí mismo.

En esa lógica, la inteligencia emocional puede convertir aspectos internos en recursos y factores protectores del ámbito interpersonal, donde la comunicación, tolerancia a la frustración y manejo emocional se convierte en aspectos fundamentales para lograr metas (Cui, 2021); por ello, es importante indagar acerca del rol que juegan las emociones en nuestra vida y cómo estas pueden convertirse en una herramienta eficaz para entablar relaciones en el campo laboral. En tal contexto, las experiencias emocionales son una parte inseparable de la vida diaria de las personas (Pekaar et al., 2020) y no es sorprendente que influyan tanto en el yo como en el otro cuando los individuos interactúan (Barreiro \& Treglown, 2020).

De igual modo, la inteligencia emocional es un factor individual que tiene una estrecha relación con la satisfacción laboral (Ouyang et al., 2015); puesto que, puede ayudar a establecer relaciones más sólidas, completar el trabajo y lograr metas personales y profesionales (Jie et al., 2021). La inteligencia emocional puede reducir el estrés y asegurar el desempeño laboral (Sadovyy et al., 2021). Por ello, la presente investigación tuvo por objetivo recopilar información en estudios desarrollados sobre la inteligencia emocional como herramienta eficaz para la mejora del desempeño laboral en funcionarios que prestan servicios en una organización. 


\section{Conceptualización de la inteligencia emocional: aproximaciones del modelo teórico}

Es importante destacar el aporte de Salovey y Mayer (1990) quienes describen la inteligencia emocional como la habilidad que se obtiene para expresar emociones y utilizarlas en el plano cognitivo, así como también comprenderlas y gestionarlas al momento de relacionarnos con los demás. De igual modo, el concepto indica que el éxito profesional individual depende tanto del conocimiento sobre uno mismo y las emociones de los demás (Mayer \& Salovey, 2007). Por su parte, Goleman (2000), atribuye a la inteligencia emocional características ligadas a involucrarnos con los demás y con nosotros mismos, denotando empatía, iniciativa, adaptación y capacidad de persuasión. En este constructo, se toman en cuenta el componente intrapersonal y el interpersonal; el primero, destaca la capacidad de control emocional, la autoconciencia y la motivación (Dueñas, 2002), mientras que, el interpersonal se caracteriza por albergar habilidades como la empatía y las habilidades sociales en sí (Goleman, 2006).

Por su parte, Baron (2006) define inteligencia emocional como el conjunto de competencias aprendidas, las que permiten a las personas interactuar, experimentar, expresar y comunicarse con los demás en diferentes demandas cotidianas. Bajo esta definición, se conceptualizan cinco dimensiones, el primero, la dimensión intrapersonal caracterizado por una conciencia de sí mismo y de los demás, por una autoestima saludable y aceptación; la dimensión interpersonal tiene como base la conciencia social, involucrado en brindar y recibir afecto (Fragoso, 2015). La dimensión manejo del estrés, relacionada con afrontar y aceptar en forma adecuada diferentes situaciones en las que el individuo subsista (Martínez et al., 2016). La dimensión adaptabilidad, hace referencia a la capacidad para manejar los problemas y buscar una solución, tomando como punto de partida lo que se siente y lo que sucede alrededor (Ugarriza, 2001). Finalmente, el componente estado de ánimo general vinculada a la autoeficacia, la cual implica que las personas poseen la capacidad de percibirse a sí misma de la forma cómo realmente desea sentirse (Fragoso, 2015).

En tal sentido, la inteligencia emocional es el manejo intencionado de las emociones con el objetivo de alcanzar comportamientos deseados en el campo laboral y obtener mejores resultados en el plano intra e interpersonal (Weisinger, 2001). De acuerdo con este modelo, la inteligencia emocional se compone de seis aspectos, cuyo primer elemento es el desarrollo de la autoconciencia que permite influir en acciones para el logro de objetivos institucionales (Carmona et al., 2015). El segundo componente, se basa en el control de emociones, para ser utilizadas a favor de alguna situación (Rico, 2002). Asimismo, el tercer componente, denominado automotivación, está 
orientado a la perseverancia de lograr una tarea (Weisinger, 2001). El cuarto componente, implica la comunicación eficaz, que pone en juego la atención dinámica, asertividad, y el trabajo en equipo (Canseco \& Ojeda, 2016). El quinto componente, se encuentra ligado a la experiencia interpersonal, orientada a generar vínculos positivos con los demás (Castelló \& Cano, 2011). Finalmente, se hace referencia al componente vinculado al apoyo a los demás, partiendo de la base de la interacción como elemento fundamental dentro del entorno laboral (Rico, 2002).

\section{Influencia de la inteligencia emocional en el desempeño laboral: análisis empírico}

Las competencias que determinan la inteligencia emocional se encuentran vinculadas al pensamiento y los sentimientos, trayendo consigo una mejora en el desempeño laboral, donde dichas aptitudes están enfocadas al aspecto personal, por determinar el dominio de sí mismo y en el aspecto social, ya que se orientan al manejo de las relaciones con otros (Fernández, 2005). Asimismo, bajo el aporte empírico de Herrera (2017) la inteligencia emocional y desempeño laboral se correlaciona y es estadísticamente significativa $(r r=.729 ; p p<.001)$. Finalmente, Ramos (2013) destaca el rol fundamental de la inteligencia emocional, al momento de establecer sinergia laboral, aseverando la necesidad de fortalecer competencias como la autoconciencia, el control emocional, la automotivación, la empatía y las relaciones interpersonales para desarrollar sinergia entre los colaboradores.

\section{Metodología}

La búsqueda bibliográfica se hizo en la base de datos de Scopus y ScienceDirect en los meses de mayo-junio del 2021, en inglés y español o castellano. Solo se consideraron artículos científicos originales, cuya publicación se hicieron en el intervalo de tiempo del 2016 al 2021. Los términos de búsqueda como palabras clave, tanto en inglés y castellano fueron los siguientes: "Inteligencia emocional" + "herramienta eficaz" + "desempeño laboral" + "funcionarios públicos"; además, se combinó entre las mismas palabras como: "Inteligencia emocional” + "desempeño laboral". De igual manera, en la búsqueda en inglés se empleó: "Emotional intelligence" + "effective tool" + "job performance" más su combinación "Emotional intelligence” + "job performance."

En la búsqueda inicial se identificaron un total de 912 artículos, hasta junio de 2021, luego de hacer un filtro por fecha y tipo de publicación se redujo a 56 artículos. Por lectura de títulos se 
redujo a 37 artículos. Por último, se revisó los resúmenes de 32 artículos según las variables de estudio, quedando 18 artículos que satisfacen el objetivo de la presente investigación; es decir se seleccionaron aquellas investigaciones que contienen a la inteligencia emocional como herramienta eficaz para la mejora del desempeño laboral.

La metodología empleada fue tomada de Sanders (2020), en cuyo estudio esquematiza y explica "cómo escribir (y cómo no escribir) un artículo de revisión científica"; así también, para determinar los criterios de búsqueda se tomó la metodología desarrollada por Danet y Jimenez, (2019) y Danet (2021). Con respecto a los términos de búsqueda, no se empleó un tesauro específico; sin embargo, los términos "inteligencia emocional”, "desempeño laboral (análisis del desempeño)" y "funcionarios públicos" con su equivalente "empleados de gobierno" se encuentran explícitos en el Tesauro DeCS - Descriptores en Ciencias de la Salud y de manera relativa, en el Tesauro Europeo de la Educación. De igual manera, se optó por realizar la búsqueda bibliográfica en la base de datos de Scopus y ScienceDirect, por la accesibilidad a dichas fuentes de información facilitadas por el Concytec.

\section{Resultados}

En este apartado, se presenta la revisión de los estudios sobre la inteligencia emocional como herramienta eficaz para la mejora del desempeño laboral en funcionarios que prestan servicios en una organización. El Gráfico1, presenta el proceso de cribado para la selección de los artículos analizados. La Tabla 1, refiere a los resultados de búsqueda en la que se indica al autor(es) y año de publicación, área temática, el tamaño de la muestra y contexto geográfico donde se realizó la investigación (país en el que se desarrolló el estudio). La Tabla 2, refiere a los resultados más significativos identificados en los artículos seleccionados. 


\section{Gráfico 1}

Proceso de cribado para la selección de los artículos a analizar

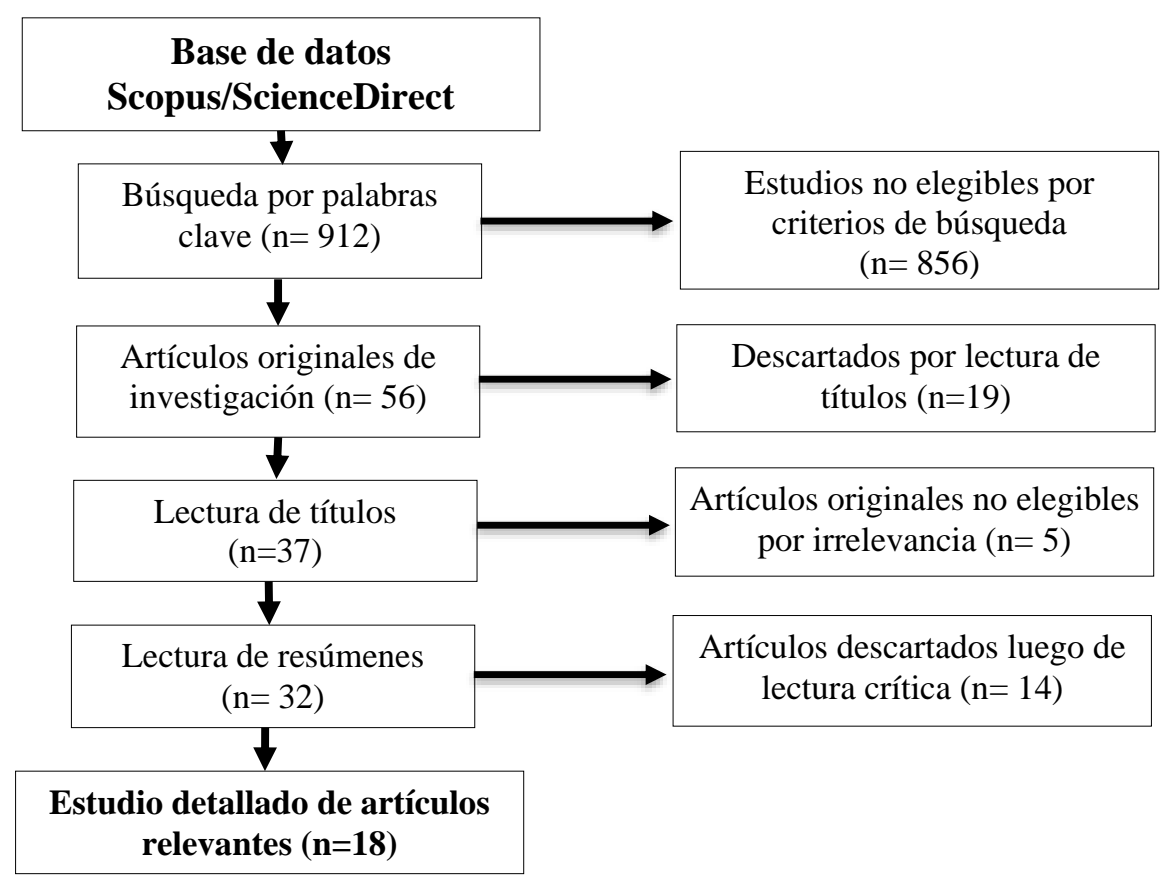




\section{Tabla 1 \\ Estudios empíricos respecto a la inteligencia emocional como herramienta eficaz para la mejora del desempeño laboral, 2016-2021}

\begin{tabular}{|c|c|c|}
\hline $\begin{array}{l}\text { Autor(es)/ año } \\
\text { de publicación }\end{array}$ & Área temática & Muestra/ Contexto geográfico \\
\hline lovyy et al. & $\begin{array}{l}\text { VID-19: Cómo el estrés generado por la pandemia puede afectar el } \\
\text { sempeño laboral a través del papel moderador de la inteligencia } \\
\text { ocional. }\end{array}$ & $\begin{array}{l}\text { s }(M=36,31, S D=13,3, \text { rango } \\
\text { ña. }\end{array}$ \\
\hline $\begin{array}{l}\text { Grover \& } \\
\text { Furnham (2021) }\end{array}$ & encia emocional y & $=9,00$, de Loirares, Kento unto y u \\
\hline Cui (2021) & $\begin{array}{l}\text { ional en la transparencia del lugar de } \\
\text { a. }\end{array}$ & Universidad de Xijing, Xi'an, China. \\
\hline $\begin{array}{l}\text { (Boadi et al., } \\
\text { 2020) }\end{array}$ & $\begin{array}{l}\text { y silencio de los empleados: la } \\
\text { inismo explicativo. }\end{array}$ & hoteles y \\
\hline $\begin{array}{l}\text { (Furnham \& } \\
\text { Taylor, 2020) }\end{array}$ & $\begin{array}{l}\text { La relación entre la inteligencia emocional y las escalas de } \\
\text { personalidad ocupacional en la alta dirección. }\end{array}$ & $\begin{array}{l}\mathrm{N}=33 \\
\text { años. Á }\end{array}$ \\
\hline $\begin{array}{l}\text { Barreiro } \\
\text { Treglown } \\
(2020)\end{array}$ & $\begin{array}{l}\text { ¿Qué hace a un empleado comprometido? Un enfoque a nivel de } \\
\text { facetas para caracterizar la inteligencia emocional como predictor del } \\
\text { compromiso. }\end{array}$ & $\begin{array}{l}\mathrm{N}=306 \text { empleados toc } \\
\text { cuales } 176 \text { eran hombr }\end{array}$ \\
\hline $\begin{array}{l}\text { Atmaca } \\
(2020)\end{array}$ & & \\
\hline $\begin{array}{l}\text { Wen } \\
(2019)\end{array}$ & $\begin{array}{l}\text { emocional, apoyo organizacional } \\
\text { un modelo de mediación moderado. }\end{array}$ & \\
\hline Prentice (2019) & Gestión de encuentros de servicio con inteligencia emocional. & \\
\hline $\begin{array}{l}\text { Alam } \\
(2019)\end{array}$ & a & $\begin{array}{l}\text { jo-familia en la } \\
\text { dio no precisa el }\end{array}$ \\
\hline $\begin{array}{l}\text { Cofer } \\
(2018)\end{array}$ & con & encia \\
\hline $\begin{array}{l}\text { Dhani \& } \\
\text { Sharma (2017) }\end{array}$ & el desempeño laboral de los & $\begin{array}{l}\mathrm{N}=157 \text { directivos de Tecnología de Información de } \\
\text { nivel medio de Delhi-NCR en la India, } 52,2 \% \\
\text { hombres y } 7547,8 \% \text { mujeres. }\end{array}$ \\
\hline $\begin{array}{l}\text { Shkoler \& } \\
\text { Tziner (2017) }\end{array}$ & $\begin{array}{l}\text { El papel mediador y moderador del burnout y la inteligencia } \\
\text { emocional en la relación entre justicia organizacional y mala conducta } \\
\text { laboral. }\end{array}$ & $\begin{array}{l}\mathrm{N}=243 \text { participantes de Israel; } 51,9 \% \text { mujeres y el } \\
48,1 \% \text { hombres, de } 20 \text { a } 60 \text { años }(\mathrm{M}=32,67, \mathrm{SD}= \\
8,87) .\end{array}$ \\
\hline $\begin{array}{l}\text { Asrar-ul-Haq } \\
\text { et al. (2017) }\end{array}$ & $\begin{array}{l}\text { Impacto de la inteligencia emocional en el desempeño docente en } \\
\text { instituciones de educación superior de Pakistán. }\end{array}$ & des de la zona \\
\hline $\begin{array}{l}\text { Clarke \& } \\
\text { Mahadi (2017) }\end{array}$ & $\begin{array}{l}\text { La importancia del respeto por el reconocimiento mutuo en la } \\
\text { mediación de las relaciones entre el rasgo de inteligencia emocional, } \\
\text { el compromiso afectivo y la satisfacción laboral. }\end{array}$ & $\begin{array}{l}\text { Los datos se recopilaron de una gran organización } \\
\text { de seguros con } 615 \text { oficinas en Malasia; encuesta } \\
\text { postal autoadministrada. }\end{array}$ \\
\hline $\begin{array}{l}\text { Bozionelos \& } \\
\text { Singh (2017) }\end{array}$ & $\begin{array}{l}\text { La relación de la inteligencia emocional con la tarea y el desempeño } \\
\text { contextual: más de lo que parece lineal. }\end{array}$ & $\begin{array}{l}\mathrm{N}=188 \text { empleados ( } 48 \text { mujeres y } 140 \text { hombres) en } \\
\text { los Emiratos Árabes Unidos. }\end{array}$ \\
\hline $\begin{array}{l}\text { Sony \& Mekoth } \\
\text { (2016) }\end{array}$ & $\begin{array}{l}\text { La relación entre la inteligencia emocional, la adaptabilidad de los } \\
\text { empleados de primera línea, la satisfacción laboral y el desempeño } \\
\text { laboral. }\end{array}$ & $\begin{array}{l}\mathrm{N}=517 \text { empleados de primera línea del país de la } \\
\text { India. }\end{array}$ \\
\hline $\begin{array}{l}\text { Mohamad } \\
\text { Jais (2016) }\end{array}$ & $\begin{array}{l}\text { Inteligencia emocional y desempeño laboral: un estudio entre maestros } \\
\text { de Malasia. }\end{array}$ & $\begin{array}{l}\text { le Kedah, localizado al noroeste } \\
\text { Malasia. }\end{array}$ \\
\hline
\end{tabular}

Nota. A partir de la Bases de datos Scpous y ScienceDirect 2016-2021. 
Tabla 2

Resultados significativos de los estudios revisados respecto a la inteligencia emocional (IE) como herramienta eficaz para la mejora del desempeño laboral, 2016-2021

\begin{tabular}{|c|c|}
\hline $\begin{array}{c}\text { Autor(es) y año de } \\
\text { publicación }\end{array}$ & Resultados significativos \\
\hline Sadovyy et al. (2021) & $\begin{array}{l}\text { Existe relación entre el estrés de Covid, el rendimiento y la inteligencia emocional. La IE ayudó a reducir el } \\
\text { estrés y aseguró el desempeño laboral. }\end{array}$ \\
\hline Grover \& Furnham (2021) & $\begin{array}{l}\text { El narcisismo, inesperadamente, no tuvo una relación significativa con el agotamiento laboral. La IE proporciona } \\
\text { un amortiguamiento para reducir el agotamiento laboral. }\end{array}$ \\
\hline Cui (2021) & $\begin{array}{l}\text { Este estudio determina que existen relaciones emocionales de inteligencia, compromiso corporativo y desempeño } \\
\text { de los empleados. }\end{array}$ \\
\hline Boadi et al. (2020) & La falta de IE de los empleados inhibe la creación conjunta de valor para el cliente con la institución. \\
\hline Furnham \& Taylor (2020) & $\begin{array}{l}\text { La IE está relacionada con diferentes formas de éxito laboral. La toma de decisiones y manejo del estrés se } \\
\text { relacionaron de manera consistente con todas las medidas de resultados laborales. }\end{array}$ \\
\hline Barreiro \& Treglown (2020) & $\begin{array}{l}\text { Los empleados con mayor felicidad disposicional, son buenos para influir en las emociones de los demás (gestión } \\
\text { de las emociones), están automotivados. }\end{array}$ \\
\hline Atmaca et al. (2020) & $\begin{array}{l}\text { Se encontraron correlaciones entre las emociones, el agotamiento y la satisfacción laboral de los docentes en } \\
\text { servicio. Las dimensiones de alegría y amor mostraron correlaciones positivas con la satisfacción laboral. }\end{array}$ \\
\hline Wen et al. (2019) & $\begin{array}{l}\text { El apoyo organizacional percibido, moderó efectivamente la mediación de la actuación profunda entre la IE y la } \\
\text { satisfacción laboral. }\end{array}$ \\
\hline Prentice (2019) & $\begin{array}{l}\text { La IE tiene un efecto significativo en la satisfacción laboral del distribuidor y la intención de rotación. La } \\
\text { satisfacción laboral media completamente el vínculo entre la IE y la intención de rotación del distribuidor. }\end{array}$ \\
\hline Alam et al. (2019) & $\begin{array}{l}\text { El estrés laboral causa un estado de ánimo negativo y hace que los empleados se preocupen cognitivamente } \\
\text { mientras están en casa. }\end{array}$ \\
\hline Cofer et al. (2018) & $\begin{array}{l}\text { No hubo asociación del agotamiento con las puntuaciones de los exámenes. Las estrategias para reducir el } \\
\text { agotamiento deben considerar enfocarse en la inteligencia emocional. }\end{array}$ \\
\hline Dhani \& Sharma (2017) & $\begin{array}{l}\text { Existe diferencias de género significativas en la inteligencia emocional y el desempeño laboral, lo que sugiere } \\
\text { que las empleadas obtienen más puntajes en IE que sus contrapartes masculinas. }\end{array}$ \\
\hline Shkoler \& Tziner (2017) & $\begin{array}{l}\text { Los hallazgos indican que la IE de hecho modera y mejora, la relación entre las percepciones de agotamiento y } \\
\text { malas conductas laborales. }\end{array}$ \\
\hline Asrar-ul-Haq et al. (2017) & $\begin{array}{l}\text { La autoconciencia emocional, la confianza en uno mismo, el logro, el desarrollo de los demás y el manejo de } \\
\text { conflictos tienen una relación positiva y significativa con el desempeño laboral. }\end{array}$ \\
\hline Clarke \& Mahadi (2017) & El respeto de reconocimiento mutuo predice la satisfacción laboral subordinada y el compromiso afectivo. \\
\hline Bozionelos \& Singh (2017) & $\begin{array}{l}\text { La IE se relaciona con el desempeño contextual y tareas en el trabajo. El entrenamiento en IE puede ser } \\
\text { beneficioso para los que obtienen puntajes promedio y no para promedios bajos. }\end{array}$ \\
\hline Sony \& Mekoth (2016) & $\begin{array}{l}\text { Existe una relación positiva entre IE y la adaptabilidad de los empleados de primera línea. La adaptabilidad de } \\
\text { empleados, tiene un impacto positivo en los resultados laborales. }\end{array}$ \\
\hline Mohamad \& Jais (2016) & $\begin{array}{l}\text { Los dominios (autoconciencia, autorregulación, automotivación, empatía y habilidades sociales) de la IE tienen } \\
\text { un mayor impacto en el rendimiento laboral de los empleados. }\end{array}$ \\
\hline
\end{tabular}

Nota. Tabla organizada a partir de la Bases de datos Scpous y ScienceDirect 2016-2021. IE: Inteligencia emocional.

\section{Discusión}

Las investigaciones anteriores a menudo han adoptado una perspectiva relativamente estática sobre la inteligencia emocional al centrarse únicamente en las diferencias individuales y sus consecuencias en diferentes ámbitos de la vida (Pekaar et al., 2020). A medida que el campo de investigación de la inteligencia emocional está madurando, los académicos se han interesado más en los mecanismos que subyacen a la manifestación de dicha variable, lo que ha resultado en 
un énfasis en el papel del contexto, la motivación y otros moderadores potenciales que influyen en el uso de la inteligencia emocional (Alam et al., 2019).

Sin embargo, hasta ahora ha faltado un marco teórico integrado que ayude a desentrañar el proceso de la inteligencia emocional a lo largo del tiempo (Udayar et al., 2020); a pesar del interés general en la inteligencia emocional, hay escasez de investigación sobre el grado en que la creación conjunta de valor para el cliente o usuario se relaciona con la inteligencia emocional de los empleados (Boadi et al., 2020). Del mismo modo, son pocos los estudios que se ocupan de la inteligencia emocional como herramienta eficaz para la mejora del desempeño laboral en funcionarios públicos (Barreiro \& Treglown, 2020). Vacíos identificados que merecen mayor atención por la academia en el presente siglo.

Por otro lado, de acuerdo a las investigaciones realizadas en este campo, el constructo inteligencia emocional influye significativamente en la mejora de las competencias laborales, alegando que el componente psicológico de cada colaborador es primordial para el fortalecimiento de sus habilidades en el lugar de trabajo (Duque et al., 2017). La inteligencia emocional conduce directa e indirectamente a reducir el agotamiento (Choi et al., 2019), o inversamente, la falta de inteligencia emocional de los empleados inhibe la creación conjunta de valor para el cliente (Boadi et al., 2020). Izquierdo y Quispitongo (2017) registraron que el desempeño laboral de los colaboradores se influencia directa y positivamente por la inteligencia emocional; a partir de ello, se recomienda la elaboración de estrategias a nivel organizacional principalmente orientado a los colaboradores.

De igual modo, Molina y Villavicencio (2012), mediante el diseño de estrategias de fortalecimiento de inteligencia emocional, demostraron que existen factores dentro del entorno laboral que pueden favorecer o no a la inteligencia emocional del personal, ello tomando en consideración la relación directa que posee esta variable con el desempeño; estos factores son vinculados a las horas de trabajo, la comunicación jerárquica, las herramientas disponibles para cumplir las funciones laborales, trabajo bajo presión ejercido y el clima organizacional; por ende, es importante la incorporación de ejercicios de relajación como la respiración diafragmática, así como también la creación de conciencia de uno mismo por medio del mindfulness.

A un nivel más usual, diversos autores llegan a concluir que la inteligencia emocional es la capacidad de una persona para controlar sus emociones, ser empática e influye en cómo interactúa con los demás (Duque et al., 2017; Mattingly \& Kraiger, 2019; Awwad et al., 2020). De igual 
modo, los hallazgos determinan que el procesamiento de las propias emociones interactúa con el procesamiento de las emociones de los demás (Pekaar et al., 2020); de modo que, los episodios emocionales son unidades de tiempo útiles para estudiar los efectos de la inteligencia emocional (Choi et al., 2019). En tal contexto, las emociones pueden expresarse hacia los demás, pueden provocar emociones en los demás o pueden ser reacciones a las emociones de los demás (Pekaar et al., 2020). Sin embargo, la falta de inteligencia emocional de los empleados inhibe la creación conjunta de valor para el cliente o usuario con la organización (Boadi et al., 2020).

Por otro lado, los estudios también revelan que los empleados con alta inteligencia emocional manejan mejor las quejas de los clientes o usuarios de una organización (Boadi et al., 2020). Por lo tanto, como las diversas organizaciones y empresas desean permanecer en el tiempo, requieren una alta inteligencia emocional de los empleados, para que puedan proponer valor en la creación conjunta del cliente (Duque, 2012; Boadi et al., 2020). En síntesis, los hallazgos puntualizan que la inteligencia emocional es una consideración vital en el lugar de trabajo y está asociada con una mayor satisfacción y desempeño laboral (Jie et al., 2021).

De igual manera, los resultados de diferentes estudios muestran una relación positiva significativa de la inteligencia emocional con la satisfacción interna o el desempeño de los colaboradores (Araujo \& Leal, 2007; Çekmecelioğlu et al., 2012; Sony \& Mekoth, 2016); así como también indican que la inteligencia emocional afecta significativamente la satisfacción laboral (Ouyang et al., 2015). Específicamente, estos hallazgos indican que los empleados con un alto reconocimiento emocional tienen más probabilidades de tener niveles más altos de satisfacción laboral (Çekmecelioğlu et al., 2012; Wen et al. 2019); regular las emociones frente a situaciones que puede ser potencialmente estresantes sobre todo en el ámbito laboral (Furnham \& Taylor, 2020).

Así también, se resalta que el rasgo de personalidad asociada a la inteligencia emocional, media la relación de la estabilidad con el desempeño laboral (Grover \& Furnham, 2021); es decir, la inteligencia emocional característica, puede entonces facilitar resultados como el desempeño de tareas (Hui-Hua \& Schutte, 2015). En consecuencia, lo alentador de las investigaciones sobre la inteligencia emocional, y como marco de contribución del presente estudio, muestra un efecto positivo en el entrenamiento de las personas en la mejora del desempeño laboral (Mattingly \& Kraiger, 2019). De modo que, una inteligencia superior lo ayudará a administrar el lugar de trabajo, los desafíos sociales, liderar e inspirar a otros y sobresalir en su carrera o trabajo (Jie et al., 2021). 
Del mismo modo, los estudios también ponen de manifiesto que los rasgos de personalidad están asociados a la inteligencia emocional, ya sea positiva o negativamente; por ejemplo, la tríada oscura (narcisismo, maquiavelismo y psicopatía) está relacionada principalmente de forma negativa con la inteligencia emocional (Michels \& Schulze, 2021); los rasgos de personalidad antisocial se asocian negativamente con el conocimiento (narcisismo) y se asocian positivamente con una mayor participación (maquiavelismo y psicopatía) (Bird et al., 2022). Los rasgos de personalidad en relación al apoyo social percibido muestran relaciones positivas con la inteligencia emocional (Di Fabio \& Saklofske, 2019); el rasgo de inteligencia emocional y el desempeño laboral se relacionan positivamente (Hui-Hua \& Schutte, 2015).

Así también, el rasgo de inteligencia emocional predice positivamente relaciones de alto respeto mutuo (Asrar-ul-Haq et al., 2017). La relación entre los rasgos de personalidad y la compasión con la autocompasión de trabajadores de organizaciones públicas, apoyan el papel mediador de la inteligencia emocional (Di Fabio \& Saklofske, 2021). Según Hui-Hua y Schutte (2015), el temperamento y los rasgos de personalidad de base biológica pueden ser la base para el desarrollo de las competencias que comprenden la inteligencia emocional.

Los resultados muestran que la inteligencia emocional está significativamente relacionada con todas las estrategias de afrontamiento, mientras que solo ciertos factores de personalidad hacen variaciones únicas (Prentice et al., 2020); es una ayuda fundamental para reducir el estrés y asegurar el desempeño laboral (Sadovyy et al., 2021). Las personas emocionalmente inteligentes son capaces de regular sus emociones para permanecer optimistas y mitigar los sentimientos negativos en el lugar de trabajo, lo que resulta en una mayor satisfacción laboral, compromiso organizacional y comportamientos de ciudadanía organizacional, así como menores conductas laborales contraproducentes e intenciones de rotación (Miao et al., 2019). Los comportamientos y competencias asociados con la regulación de las emociones, la adherencia a los objetivos y las interacciones positivas con los demás, conducen a un mejor desempeño (Hui-Hua \& Schutte, 2015).

El marco de revisión, en primer lugar, puede contribuir con una visión más completa de la importancia de la inteligencia emocional en el desempeño laboral de las personas cuando estas tienen un control sobre sus emociones, puesto que, puede funcionar como parte de un proceso emocional que se puede activar, intervenir o construir. En segundo lugar, los hallazgos en el campo de la inteligencia emocional, puede inspirar a los investigadores a integrar los estímulos que activan 
el procesamiento de las emociones en sus modelos de investigación; por último, dicho estudio ofrece perspectivas que le permite innovar al investigador sobre la dinámica de la vida emocional que describe nuestro marco y llevarlo a otros contextos.

Finalmente, en cuanto a las limitaciones, se evaluaron estudios desarrollados sobre la inteligencia emocional como herramienta eficaz para la mejora del desempeño laboral en funcionarios que prestan servicios en una organización. Por lo tanto, la generalización de los hallazgos se limita a individuos sujetos a desafíos y condiciones de desempeño similares. De igual modo, el estudio no hace una caracterización detallada de las dimensiones de la inteligencia emocional, lo que puede representar una captura incompleta del constructo de la inteligencia emocional; además, la revisión solo se realizó en dos bases de datos, lo que pudieron pasar desapercibidos otros resultados con grado de contribución; sin embargo, esto puede dar origen a construir un marco actual más completo en cada una de las dimensiones de la inteligencia emocional. Por otro lado, se reconoce que no se priorizó una escala de tiempo aún más detallada, entre la evaluación de la inteligencia emocional, regulación de las emociones y el desempeño laboral, de modo que, puede inspirar a los investigadores a identificar posibles microprocesos que operan a favor de dichas variables.

\section{Conclusiones}

Tomando en cuenta los aportes teóricos que definen la inteligencia emocional, se concluye que es la capacidad para entender y comprender las emociones propias y la de los demás, tomando en cuenta procesos vinculados al autoconocimiento, así como también, la empatía y asertividad, ya que se encuentra basada en un enfoque centrado en el individuo y su búsqueda de independencia e interacción social. En el manejo de conflictos, encuentra un equilibrio entre la satisfacción de necesidades como validación y obtención de independencia.

Con respecto a la literatura indagada y las investigaciones, es preciso mencionar la relación directa y significativa entre la inteligencia emocional y el desempeño laboral, ello indica el rol fundamental que poseen las competencias blandas de un colaborador; tal es así, que la inteligencia emocional se ha posicionado como uno de los elementos más indispensables dentro del entorno laboral, pues no solo permite mantener motivado al personal, sino también mejora el clima laboral y faculta al funcionario interactuar saludablemente con sus superiores y colegas. Por último, la 
inteligencia emocional se ha relacionado con sólidos parámetros de desempeño laboral, como el liderazgo, y es un atributo importante a tener en cuenta en funcionarios públicos.

\section{Referencias}

Alam, M., Ezzedeen, S. R., \& Latham, S. D. (2019). Managing work-generated emotions at home: An exploration of the "Bright Side" of emotion regulation. Human Resource Management Review, 29 (4), 100678. https://doi.org/10.1016/j.hrmr.2018.12.002

Araujo, M. \& Leal, M. (2007). Inteligencia emocional y desempeño laboral en las instituciones de educación superior públicas. Universidad Rafael Belloso Chacín.

Asrar-ul-Haq, M., Anwar, S., \& Hassan, M. (2017). Impact of emotional intelligence on teacher's performance in higher education institutions of Pakistan. Future Business Journal, 3 (2), 87-97. https://doi.org/10.1016/j.fbj.2017.05.003

Atmaca, Ç., Rızaoğlu, F., Türkdoğan, T., \& Yaylı, D. (2020). An emotion focused approach in predicting teacher burnout and job satisfaction. Teaching and Teacher Education, 90. https://doi.org/10.1016/j.tate.2020.103025

Awwad, D. A., Lewis, S. J., Mackay, S., \& Robinson, J. (2020). Examining the Relationship between Emotional Intelligence, Leadership Attributes and Workplace Experience of Australian Chief Radiographers. Journal of Medical Imaging and Radiation Sciences, 51 (2), 256-263. https://doi.org/10.1016/j.jmir.2020.01.002

Baron, R. (2006). El modelo de Bar-On de Inteligencia Emocional-Social (ESI). Psicothema, 18 (1), 13-25. http://www.psicothema.com/pdf/3271.pdf

Barreiro, C. A., \& Treglown, L. (2020). What makes an engaged employee? A facet-level approach to trait emotional intelligence as a predictor of employee engagement. Personality and Individual Differences, 159 (2019), 1-13. https://doi.org/10.1016/j.paid.2020.109892

Bird, R. T., Hickey, H. K., Leavitt, M. J., \& Robinson, J. L. (2022). The dark authoritarians: Profiling the personality, emotional style, and authoritarian attitudes of the major American parties. Personality and Individual Differences, 186 (PB), 111298.

https://doi.org/10.1016/j.paid.2021.111298

Boadi, E. A., He, Z., Boadi, E. K., Antwi, S., \& Say, J. (2020). Customer value co-creation and employee silence: Emotional intelligence as explanatory mechanism. International Journal of Hospitality Management, 91 (2019). https://doi.org/10.1016/j.ijhm.2020.102646

Bozionelos, N., \& Singh, S. K. (2017). The relationship of emotional intelligence with task and contextual performance: More than it meets the linear eye. Personality and Individual Differences, 116, 206-211. https://doi.org/10.1016/j.paid.2017.04.059 
Canseco, F. \& Ojeda, A. (2016). Comunicación laboral: una propuesta estratégica para facilitar el quehacer de los trabajos en equipo. Enseñanza e Investigación en Psicología, 21 (2), 183194. https://www.redalyc.org/pdf/292/29248181009.pdf

Carmona, P.; Vargas, J. \& Rosas, R. (2015). Influencia de la inteligencia emocional en el desempeño laboral. Sapienza Organizacional, 2 (3), 53-68. https://bit.ly/3cUKvWr

Castelló, A. \& Cano, M. (2011). Inteligencia interpersonal: conceptos clave. Revista Electrónica Interuniversitaria de Formación del Profesorado, 14 (3), 23-35. https://www.redalyc.org/pdf/2170/217022109002.pdf

Çekmecelioğlu, H. G., Günsel, A., \& Ulutaş, T. (2012). Effects of Emotional Intelligence on Job Satisfaction: An Empirical Study on Call Center Employees. Procedia - Social and Behavioral Sciences, 58, 363-369. https://doi.org/10.1016/j.sbspro.2012.09.1012

Choi, H. M., Mohammad, A. A. A., \& Kim, W. G. (2019). Understanding hotel frontline employees' emotional intelligence, emotional labor, job stress, coping strategies and burnout. International Journal of Hospitality Management, 82 (May), 199-208. https://doi.org/10.1016/j.ijhm.2019.05.002

Clarke, N., \& Mahadi, N. (2017). The significance of mutual recognition respect in mediating the relationships between trait emotional intelligence, affective commitment and job satisfaction. Personality and Individual Differences, 105, 129-134. https://doi.org/10.1016/j.paid.2016.09.028

Cofer, K. D., Hollis, R. H., Goss, L., Morris, M. S., Porterfield, J. R., \& Chu, D. I. (2018). Burnout is Associated With Emotional Intelligence but not Traditional Job Performance Measurements in Surgical Residents. Journal of Surgical Education, 75 (5), 1171-1179. https://doi.org/10.1016/j.jsurg.2018.01.021

Cui, Y. (2021). The role of emotional intelligence in workplace transparency and open communication. Aggression and Violent Behavior, March, 101602. https://bit.ly/3qhI6L5

Danet, A. (2021). Impacto psicológico de la COVID-19 en profesionales sanitarios de primera línea en el ámbito occidental. Una revisión sistemática. Medicina Clínica, 156 (9), 449-458. https://doi.org/10.1016/J.MEDCLI.2020.11.009

Danet, A., \& Jimenez, P. M. (2019). Vivencias y emociones profesionales en el proceso de donación y trasplantes de órganos. Una revisión sistemática. Cirugía Española, 97 (7), 364376. https://doi.org/10.1016/J.CIRESP.2019.01.015

Dhani, P., \& Sharma, T. (2017). Effect of Emotional Intelligence on Job Performance of IT employees: A gender study. Procedia Computer Science, 122, 180-185. https://doi.org/10.1016/j.procs.2017.11.358

Di Fabio, A., \& Saklofske, D. H. (2019). Positive relational management for sustainable development: Beyond personality traits-the contribution of emotional intelligence. 
Sustainability (Switzerland), 11 (2), 1-9. https://doi.org/10.3390/su11020330

Di Fabio, A., \& Saklofske, D. H. (2021). The relationship of compassion and self-compassion with personality and emotional intelligence. Personality and Individual Differences, 169(May 2020), 110109. https://doi.org/10.1016/j.paid.2020.110109

Dueñas, M. (2002). Importancia de la inteligencia emocional: un nuevo reto para la orientación educativa. Educación XX1, 1 (5), 77- 96. https://www.redalyc.org/pdf/706/70600505.pdf

Duque, J. (2012). Emociones e inteligencia emocional: Una aproximación a su pertinencia y surgimiento enlas organizaciones. Revista Libre Empresa, 9 (2), 147-169.

Duque, J.; García, M. \& Hurtado, A. (2017). Influencia de la inteligencia emocional sobre las competencias laborales: un estudio empírico con empleados del nivel administrativo. Estudios Gerenciales, 33 (1), 250-260. http://dx.doi.org/10.1016/j.estger.2017.06.005

Fernández, J. (2005). Gestión por competencias. Pearson Educación S.A.

Fragoso, R. (2015). Inteligencia emocional y competencias emocionales en educación superior, ¿un mismo concepto? Revista Iberoamericana de Educación Superior, 6 (16), 110-125. https://www.redalyc.org/pdf/2991/299138522006.pdf

Furnham, A., \& Taylor, N. (2020). The relationship between emotional intelligence and occupational personality scales in senior management. Personality and Individual Differences, 154 (February 2019), 109647. https://doi.org/10.1016/j.paid.2019.109647

Goleman, D. (2000). La práctica de la inteligencia emocional. Editorial Kairos.

Goleman, D. (2006). Inteligencia social. La nueva ciencia de las relaciones. Editorial Kairós.

Grover, S., \& Furnham, A. (2021). Does emotional intelligence and resilience moderate the relationship between the Dark Triad and personal and work burnout? Personality and $\begin{array}{llll}\text { Individual Differences, } & 169 & \text { (March } & \text { 2020), }\end{array}$ https://doi.org/10.1016/j.paid.2020.109979

Herrera, G. (2017). La inteligencia emocional y su relación con el desempeño laboral de los colaboradores de la municipalidad provincial de San Martín, Región San Martín. Universidad Peruana Unión.

Hui-Hua, Z., \& Schutte, N. S. (2015). Personality, emotional intelligence and other-rated task performance. Personality and Individual Differences, 87, 298-301. https://doi.org/10.1016/j.paid.2015.08.013

Izquierdo, G. \& Quispitongo, E. (2017). Influencia de la inteligencia emocional sobre el desempeño laboral percibido en vendedores de telefonía móvil, Perú 2017. Universidad San Ignacio de Loyola. https://bit.ly/3qfuRdQ

Jie, H., Martínez, O. S., \& Crespo, R. G. (2021). The influence of employee emotional intelligence on enterprise innovation performance using an adaptive mathematical modeling of 
emotions. Aggression and Violent Behavior, (9), 101538. https://doi.org/10.1016/j.avb.2020.101538

Macht, G. A., Nembhard, D. A., \& Leicht, R. M. (2019). Operationalizing emotional intelligence for team performance. International Journal of Industrial Ergonomics, 71 (2), 57-63. https://doi.org/10.1016/j.ergon.2019.02.007

Martínez, J.; García, A. \& Aldebarán, V. (2016). Estrategias para el control del estrés empresarial. Un estudio longitudinal en una empresa mediana. Horizonte sanitario, 16 (1), 38-45. https://doi.org/10.19136/hs.v16i1.1388.

Mattingly, V., \& Kraiger, K. (2019). Can emotional intelligence be trained? A meta-analytical investigation. Human Resource Management Review, 29 (2), 140-155. https://doi.org/10.1016/j.hrmr.2018.03.002

Mayer, J. D., \& Salovey, P. (2007). ¿Qué es la inteligencia emocional? En J. D. Mayer \& D. J. Sluyter (Eds.), Desarrollo emocional e inteligencia emocional (pp. 3-31). Basic Books.

Miao, C., Humphrey, R. H., Qian, S., \& Pollack, J. M. (2019). The relationship between emotional intelligence and the dark triad personality traits: A meta-analytic review. Journal of Research in Personality, 78, 189-197. https://doi.org/10.1016/j.jrp.2018.12.004

Michels, M., \& Schulze, R. (2021). Emotional intelligence and the dark triad: A meta-analysis. Personality and Individual Differences, 180 (4), 110961.

https://doi.org/10.1016/j.paid.2021.110961

Mohamad, M., \& Jais, J. (2016). Emotional Intelligence and Job Performance: A Study among Malaysian Teachers. Procedia Economics and Finance, 35 (10), 674-682. https://doi.org/10.1016/s2212-5671(16)00083-6

Molina, M \& Villavicencio, O. (2012). Estrategias de inteligencia emocional aplicada a la empresa distribuidora CASAVAL y su impacto en el desempeño laboral. Universidad Politécnica Salesiana. https://bit.ly/3vGKVX3

Ouyang, Z., Sang, J., Li, P., \& Peng, J. (2015). Organizational justice and job insecurity as mediators of the effect of emotional intelligence on job satisfaction: A study from China. Personality and Individual Differences, 76, 147-152. https://bit.ly/3xIb6y6

Pekaar, K. A., van der Linden, D., Bakker, A. B., \& Born, M. P. (2020). Dynamic self- and otherfocused emotional intelligence: A theoretical framework and research agenda. Journal of Research in Personality, 86, 103958. https://doi.org/10.1016/j.jrp.2020.103958

Prentice, C., Zeidan, S., \& Wang, X. (2020). Personality, trait EI and coping with COVID 19 measures. International Journal of Disaster Risk Reduction, 51 (7), 101789. https://doi.org/10.1016/j.ijdrr.2020.101789

Prentice, D. C. (2019). Managing service encounters with emotional intelligence. Journal of Retailing and Consumer Services, 51 (7), 344-351. https://bit.ly/3wH0P4U 
Ramos, A. (2013). La inteligencia emocional como herramienta para desarrollar sinergia laboral en los empleados de la alcaldía municipal de Ilopango. (Tesis de pregrado; Universidad de El Salvador). http://ri.ues.edu.sv/id/eprint/4780

Rico, C. (2002). La inteligencia emocional en el trabajo de Hendrie Weisinger. Revista Facultad de Ciencias Económicas: Investigación y Reflexión, 10 (2), 101-104. https://www.redalyc.org/pdf/909/90910213.pdf

Sadovyy, M., Sánchez-Gómez, M., \& Bresó, E. (2021). COVID-19: How the stress generated by the pandemic may affect work performance through the moderating role of emotional intelligence. Personality and Individual Differences, $180 \quad$ (3), 110986. https://doi.org/10.1016/j.paid.2021.110986

Salovey, P. \& Mayer, J. (1990). Emotional intelligence. Imagination, Cognition and Personality, 9 (1), 185-211. https://doi.org/10.2190/DUGG-P24E-52WK-6CDG

Sanders, D. A. (2020). How to write (and how not to write) a scientific review article. Clinical Biochemistry, 86, 67-67. https://doi.org/10.1016/J.CLINBIOCHEM.2020.10.001

Shkoler, O., \& Tziner, A. (2017). El papel mediador y moderador del burnout y de la inteligencia emocional en la relación entre la justicia organizacional y el mal comportamiento en el trabajo. Revista de Psicología del Trabajo y de las Organizaciones, 33 (2), 157-164. https://doi.org/10.1016/j.rpto.2017.05.002

Sony, M., \& Mekoth, N. (2016). The relationship between emotional intelligence, frontline employee adaptability, job satisfaction and job performance. Journal of Retailing and Consumer Services, 30, 20-32. https://doi.org/10.1016/j.jretconser.2015.12.003

Udayar, S., Fiori, M., \& Bausseron, E. (2020). Emotional intelligence and performance in a stressful task: The mediating role of self-efficacy. Personality and Individual Differences, 156 (12). https://doi.org/10.1016/j.paid.2019.109790

Ugarriza, N. (2001). La evaluación de la inteligencia emocional a través del inventario de BarOn (I-CE) en una muestra de Lima Metropolitana. Persona, 1 (4), 129-160. https://www.redalyc.org/pdf/1471/147118178005.pdf

Weisinger, H. (2001). La inteligencia emocional en el trabajo. Suma de Letras.

Wen, J., Huang, S. (Sam), \& Hou, P. (2019). Emotional intelligence, emotional labor, perceived organizational support, and job satisfaction: A moderated mediation model. International Journal of Hospitality Management, $81 \quad$ (12), $120-130$. https://doi.org/10.1016/j.ijhm.2019.01.009 